\title{
Perspectivas del Derecho a la Ciudad en Puebla ${ }^{1}$
}

\author{
Perspectives of the Right to the City in Puebla \\ Claudia Guadalupe, De la Fuente Morales²; Fernando, Méndez Sánchez ${ }^{3}$
}

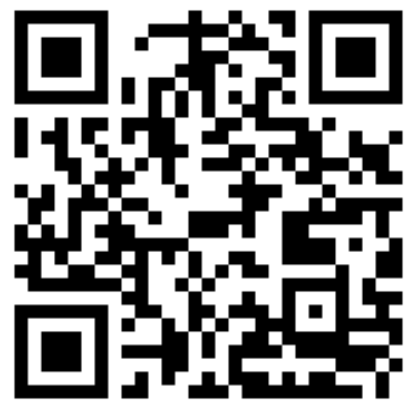

Fecha de recibido: $16-12-2020$

Fecha de aceptado: 21-02-2021

\section{(cc) BY-NC-ND}

Esta obra está bajo una licencia de Creative Commons Reconocimiento-NoComercialSinObraDerivada 4.0 Internacional

\section{RESUMEN}

El denominado Derecho a la Ciudad, parte de la premisa de que la población pueda recuperar los espacios urbanos que originalmente les corresponden y hacerlos nuevamente parte de su propia cultura, independientemente de los intereses del capitalismo salvaje. Desde el punto de vista de este derecho, se propuso un estudio de la Ciudad de Puebla, analizando su fundación, y la manera en que la población ha sufrido una planificación territorial muy específica, de la cual se desprenden ahora muchos de los problemas de violencia y conflictos sociales que se viven al interior de esta. Se utilizaron los métodos inductivo, histórico y analítico, recurriendo a instrumentos nacionales e internacionales, con especial énfasis en estos últimos. Dentro de las principales conclusiones que se obtuvieron se debe destacar la necesidad de aplicar un protocolo para coadyuvar con el desarrollo del Derecho a la Ciudad en Puebla, que abarque tres aspectos: comunidad, delincuencia y ambiente.

Palabras claves: Derecho a la Ciudad, derechos económicos-sociales y culturales, Puebla de los Ángeles.

\begin{abstract}
The so-called Right to the City, starts from the premise that the population can recover the urban spaces that originally corresponded to them and make them part of their own culture again, independently of the interests of savage capitalism. From the point of view of this right, a study of the City of Puebla is proposed, showing how since its foundation, it has been part of an elite plan in which the population has been directed to a very specific territorial planning, of the which now give rise to many of the problems of violence and social conflicts that are experienced within it. Inductive, historical, and analytical methods were used, using national and international instruments, with special emphasis on the latter. Among the main conclusions reached, we should highlight the need to apply a protocol that contributes to the development of the Right to the City in Puebla, covering three aspects: community, crime and environment.
\end{abstract}

Keywords: Economic-Social and Cultural Rights, Puebla de los Ángeles, right to the city.

Cómo referenciar este artículo:

De la Fuente., M., C., \& Méndez., S., F. (2021). Perspectivas del Derecho a la Ciudad en Puebla. Revista Politica Globalidad y Ciudadanía, 7(14), 84-102. https://doi.org/10.29105/pgc7.14-5

\footnotetext{
${ }^{1}$ Este artículo es producto del proyecto de investigación Derecho a la Ciudad financiado por la Benemérita Universidad Autónoma del Estado de Puebla y la Universidad Popular Autónoma del Estado de Puebla. Iniciado en 2019 y finalizado en 2020.

${ }^{2}$ Benemérita Universidad Autónoma de Puebla, México. Doctora en Economía Política del Desarrollo, Profesora-investigadora de la Facultad de Derecho y Ciencias Sociales de la Benemérita Universidad Autónoma de Puebla, Email: clausita_16@hotmail.com, Orcid: http://orcid.org/0000-0001-8254-4359.

${ }^{3}$ Universidad Popular Autónoma del Estado de Puebla, México. Doctor en Derecho, Director de la Facultad de Derecho de la Universidad Popular Autónoma del Estado de Puebla, Email: fms291077@gmail.com, Orcid: https://orcid.org/0000-0002-9819-9593
} 


\section{1.- INTRODUCCIÓN}

El Derecho a la Ciudad, por su parte, establece en términos generales la prerrogativa de los habitantes de toda urbe sobre decidir y crear la ciudad, y hacer de esta un espacio común en el que todos tengan acceso a bienes y servicios, decidiendo por ellos y el beneficio colectivo, en lugar de intereses individuales que puedan verse más fácilmente reflejados por el peso del capital.

La Ciudad de Puebla de los Ángeles, o Puebla de Zaragoza en la actualidad, presenta severos problemas que se ven agraviados por la falta de acceso de diversos sectores de la población, a una correcta distribución de servicios y atención de dichos problemas. Dos de ellos, típicos de todo núcleo urbano, pero particularmente acentuados en la Angelópolis, son probables derivaciones de no atender lo que hoy en día se conoce como Derecho a la Ciudad. Los contrastes son severos, especialmente en el acceso de áreas verdes que se encuentran localizados en zonas específicas de la urbe, que hacen que gran parte de la población que vive en zonas de notorio hacinamiento, tenga que trasladarse varios kilómetros para gozar de un poco de espacio libre y aire limpio. El problema de la delincuencia, por otra parte, no respeta ya zonas, además de que vecinos de diversas zonas y calles de la ciudad, opten por cerrar las mismas, impidiendo el derecho básico de tránsito, en un interés legítimo, pero no legalmente aplicado, de protegerse de la delincuencia. Por lo anteriormente señalado, cabe establecer la siguiente pregunta rectora: ¿Cómo puede establecerse un Derecho a la Ciudad en Puebla?

Para responder a la pregunta rectora planteada, es necesario establecer como objetivo del presente estudio, el fundamentar mediante propuestas teóricas referentes al Derecho a la ciudad, la validez de implementar instrumentos sociales que mitiguen los problemas que afectan a la ciudad.

Cabe señalar que el presente trabajo es solamente una primera aproximación teórica, siendo esta la primera parte de un proyecto a implementarse más adelante en convenio con instituciones de educación superior.

\section{Concepto y generalidades del Derecho a la Ciudad}

Henri Lefebvre define el Derecho a la ciudad como el "derecho de los habitantes urbanos a construir, decidir y crear la ciudad, y hacer de esta un espacio privilegiado de lucha anticapitalista” (Molano, 2016, 4). En este sentido, es necesario señalar que su constructo ideológico fue inmediatamente de la mano con 
De la Fuente., M., C., \& Méndez., S., F.

los Derechos Económicos y Sociales, ya que su propuesta se centró en los grupos vulnerables y su capacidad de crear y recuperar la ciudad. Algunos autores incluso señalan su propuesta como un nuevo humanismo; sin embargo, su teoría fue meramente "anticapitalista" que, si bien fue adecuada para los movimientos que observó en la Francia de su tiempo, ha sido ya superada por postulados de la corriente crítica, en la que lo económico es uno de muchos factores sociales que intervienen en la conducta y las relaciones entre los grupos que conforman a la sociedad. Ejemplo de las nuevas propuestas teóricas es el postulado de Laurence Costes $(2014,4)$ quien considera que la institucionalización del derecho a la ciudad es una oportunidad abierta para las luchas urbanas de retomar la protección de los derechos civiles y humanos, que se habían quedado exclusivamente en el discurso político.

David Harvey, como otro teórico que aportó a la teoría del Derecho a la Ciudad, señaló que se debía basar en lo que denominó prácticas ciudadanas emergentes (Molano, 2016, 8). Edward Soja superó el proyecto teórico de Lefebvre, incluyendo nuevas dimensiones que no se mantienen en lo puramente urbano, sino en la manera en la que la clase, el género, la etnicidad y la cultura, provocan una diversidad de formas de experimentar la ciudad adicionando a la teoría del Derecho a la Ciudad una mirada “ecléctica” (Molano, 2016, 11). Por su parte, Patrice Mele señaló que los conflictos urbanos pueden ser calificados de luchas por la tierra y los servicios urbanos en oposición a proyectos públicos o a la autorización de ciertos usos urbanos.

Al igual que en su momento Soja, Patrice Mele $(2016$, 128) acusó que la presencia de movimientos sociales como marchas o plantones, en la que los vecinos movilizados constituyen redes de militancia, son en realidad la expresión de reivindicar abusos sociales, ambientales y de acceso a servicios públicos que debía considerarse para preservan la calidad de vida.

Pero pese a lo anterior, un elemento que se ha presentado como elusivo a los principales teóricos del Derecho a la Ciudad y que ha sido tratado de manera superficial, cuando en realidad es un factor preponderante y en el que también se conjugan los Derechos Sociales y Culturales, es el que se vincula con el Derecho a la Salud.

En correspondencia con la salud, se encuentra un vínculo poco abordado desde la teoría, y aún más desde la práctica, que es la relación entre el incremento en la violencia y el decrecimiento de áreas verdes que se atenderá más adelante. 
En el ámbito internacional, es esencial señalar el fundamento normativo del Derecho a la Ciudad desde la perspectiva del Derecho Internacional de los Derechos Humanos, que abarca la creación de la Organización de las Naciones Unidas, con la respectiva Declaración Universal de los Derechos humanos, el Pacto Internacional de Derechos Económicos, Sociales y Culturales, finalizando con la denominada Carta Mundial por el Derecho a la Ciudad.

\section{Breve bosquejo de la evolución histórica del Derecho a la Ciudad}

El término de "Derecho a la Ciudad” apareció en 1968, cuando el francés Henri Lefebvre escribió el libro del mismo nombre tomando en cuenta el impacto negativo sufrido por las ciudades en los países de economía capitalista, con la conversión de la ciudad en una mercancía al servicio exclusivo de los intereses de la acumulación del capital. Como contrapropuesta a este fenómeno, Lefebvre construye una propuesta política que parte de la ciudad para reivindicar la posibilidad que la gente volviera a ser dueña del espacio en el que habitaba, oponiéndose a los efectos del neoliberalismo, entre los que se podían señalar la privatización de los espacios urbanos, el uso mercantil de la ciudad, la predominancia de industrias y espacios comerciales, así como zonas residenciales con determinadas preferencias para gozar de servicios públicos de mejor calidad.

El Derecho a la Ciudad propone una nueva perspectiva política, considerando que la urbe, particularmente en el siglo XX, dejó de pertenecer a la gente. Lefebvre aboga entonces por "rescatar el hombre como elemento principal, protagonista de la ciudad que él mismo ha construido". El derecho a la ciudad es entonces restaurar el sentido de ciudad, instaurar la posibilidad del "buen vivir" para todos, y hacer de la ciudad "el escenario de encuentro para la construcción de la vida colectiva" (Mathivet, 2009). Su propuesta tenía una poderosa influencia de corte socialista, recurriendo a autores tales como Engels y Marx, y recurriendo a instrumentos teóricos relativos a la acumulación del capital y los monopolios, pero aún más importante, partió de los mismo para el neologismo de las isotopías como tendencia a la uniformización y control capitalista del espacio que se opone conceptualmente a las heterotopías, es decir, las diversas maneras de ocupar el espacio en resistencia al capital (Molano, 2016).

El siguiente paso en la maduración del concepto del Derecho a la Ciudad, lo dio David Harvey (2008), presionando los límites de las conceptualizaciones marxistas con su reivindicación de las prácticas ciudadanas insurgentes, que son resultado de la supresión de ideales de identidad urbana y pertenencia, en los que incluso la idea de que la ciudad pueda funcionar como cuerpo político colectivo, es poco probable. Derivado de ello, Harvey denuncia la existencia de movimientos sociales urbanos que 
De la Fuente., M., C., \& Méndez., S., F.

intentan superar el aislamiento y remodelar la ciudad con una imagen diferente de la promovida por los intereses neoliberales. Harvey considera que la expansión geográfica y la reorganización espacial de los intereses neoliberales, es la que crea nuevas instalaciones de capital fijo, como puertos, aeropuertos, carreteras e hidroeléctricas; y a medida que se expande geográficamente, el capital incrementa las dinámicas de construcción y destrucción espacial, lo que explica el incremento de la violencia y el despojo de personas de áreas urbanas y semiurbanas.

Así, Harvey aspira a que las prácticas insurgentes de los sujetos sociales urbanos puedan permitir la creación de entornos no controlados por el capital, sino por el bien común, es decir, aquellas que ejemplifican la vida ya existente en plazas y jardines, basadas en la colaboración y que preceden a las teorías o paradigmas de ciudad tales como teatros comunitarios, huertos urbanos colectivos, mercados populares que se convierten en "metáforas vivas del concepto práctica ciudadana" (Gutiérrez, 2016). "Harvey plantea el derecho a la ciudad como una estación de paso [...] en perspectiva de un orden socioespacial no capitalista” (Molano, 2016).

Sería labor del geógrafo Edward Soja, el empujar el Derecho a la ciudad más allá de las conceptualizaciones puramente marxistas, incluyendo a las clases socioeconómicas, al género, a la etnicidad y las expresiones culturas, como factores que afectan la manera en la que se experimenta la ciudad; incluyó elementos y estudios de género, partiendo inicialmente del feminismo y propuso además, pasar de la teoría a la práctica a través de las universidades y servicios educativos (Soja, 2014, 211-215). Soja señala cómo el mundo está compuesto de "capas de organización macroespacial" resultado de lo económico, administrativo, cultural y político, en las que la dominación y el control social, han derivado en divisiones globales tales como las de primer y tercer mundo, que inciden en las actividades diarias de los individuos y colectividades, y que tarde o temprano pueden derivar en explosivos levantamientos sociales tales como los que se dieron en Los Ángeles en 1992 o en París en 2005; dichos disturbios, acusa Soja, surgen cuando la privación y las injusticias, enterradas bajo geografías irrumpen a la superficie, exponiendo estructuras de privilegios basados en la raza, la clase, el género y otras formas de discriminación y opresión social (Soja, 2014, 68-69).

En este sentido, Molano (2016) señala sobre Soja que su teoría del Derecho a la Ciudad se basa en tres pilares: la construcción social del espacio, la ciudad entendida como posmetrópolis y las luchas por la justicia espacial y la democracia regional, en los que la difusión de una mayor sensibilidad hacia 
las cuestiones interculturales, de etnia y de género son el punto de partida para lograr una democratización del espacio urbano.

Un paso fundamental en la construcción del Derecho a la Ciudad ha sido la elaboración de la Carta Mundial por el Derecho a la Ciudad articulada por Habitat International Coalition (HIC). Un conjunto de movimientos populares, organizaciones no gubernamentales, asociaciones profesionales, foros y redes nacionales e internacionales de la sociedad civil, comprometidas con las luchas sociales por ciudades justas, democráticas, humanas y sustentables, construyeron una Carta Mundial por el Derecho a la Ciudad que busca recoger los compromisos y medidas que deben ser asumidos por la sociedad civil, los gobiernos locales y nacionales, parlamentarios y organismos internacionales para que todas las personas vivan con dignidad en las ciudades (Mathivet, 2009). La Carta establece desde su primer artículo cinco puntos principales del Derecho a la Ciudad (UCLG, 2014):

1. Todas las personas tienen derecho a la ciudad sin discriminaciones, preservando su memoria e identidad cultural.

2. La urbe es un espacio colectivo culturalmente rico y diversificado que pertenece a todos sus habitantes.

3. Las ciudades y las autoridades deben buscar de manera progresiva la plena efectividad de los derechos económicos, sociales, culturales y ambientales, utilizando instrumentos legislativos.

4. Se considera urbe a "toda villa, aldea, capital, localidad, suburbio, ayuntamiento o pueblo que esté organizado institucionalmente como unidad local de gobierno de carácter Municipal o Metropolitano, tanto sea urbano, semirural o rural".

5. Se estima como ciudadanos y ciudadanas a "todas las personas que habiten en forma permanente o transitoria en las ciudades".

\section{2.- FUNDAMENTO NORMATIVO}

La relación del Derecho a la Ciudad con los Derechos económicos, Sociales y Culturales (DESC)

Los Derechos Económicos, Sociales y Culturales (DESC) están considerados como la segunda generación de Derechos Humanos que se ha ido incorporando en la normatividad a finales del siglo XX, su función principal es garantizar condiciones de vida dignas para todos, por lo que el valor principal que defiende es la igualdad. Los DESC son derechos relativos a las condiciones económicas y sociales que 
De la Fuente., M., C., \& Méndez., S., F.

buscan cubrir las necesidades básicas de las personas como son trabajo, educación, vivienda, seguridad social, salud, alimentación, medio ambiente sano, entre otros.

En el marco del Derecho Internacional de los Derechos Humanos, se encuentran tres normatividades base que proporcionan un marco común y que establecen obligaciones para el Estado, a saber, la Declaración Universal de los Derechos Humanos (DUDH), el Pacto Internacional de Derechos Civiles y Políticos (PIDCP) y el Pacto Internacional de Derechos Económicos, Sociales y Culturales (PIDESC). Estos marcos ofrecen una herramienta esencial para la promoción y respeto de los Derechos Humanos (DDHH), que permitirán la conducción hacia la justicia, el bienestar social y la igualdad.

Se debe destacar que el principio de progresividad es esencial para el goce de los DESC, todos los Estados deben utilizar sus recursos, medidas para lograr el respeto y la efectividad de los derechos económicos, sociales, culturales y ambientales.

Artículo 2. 1. Cada uno de los Estados Parte en el presente Pacto se compromete a adoptar medidas, tanto por separado como mediante la asistencia y la cooperación internacionales, especialmente económicas y técnicas, hasta el máximo de los recursos de que disponga, para lograr progresivamente, por todos los medios apropiados, inclusive en particular la adopción de medidas legislativas, la plena efectividad de los derechos aquí reconocidos (Office of the United Nations High Commissioner for Human Rights -OHCHR), 1966).

Es inaceptable que, bajo esta premisa en lugar de avanzar se tenga un retroceso en la plena efectividad de los DESC y se observen personas sin empleos, sin seguridad social, viviendo en zonas de riesgo en un ambiente que no es sano, por lo que es "obligación de las autoridades para cumplir con los mínimos indispensables de estos derechos es ineludible" (Comisión Nacional de los Derechos Humanos (CNDH), 2019).

En este sentido la relación entre los DESC y el Derecho a la Ciudad se da en torno a la vida colectiva, es buscar el pleno goce de derechos desde una visión integradora, para poder vivir dignamente, “el derecho a la ciudad es la posibilidad de construir una ciudad en la que se pueda vivir dignamente, reconocerse como parte de ella, y donde se posibilite la distribución equitativa de diferentes tipos de recursos: trabajo, de salud, de educación, de vivienda, recursos simbólicos: participación, acceso a la información, etc.” (Mathivet, 2009).

90 Revista Política, Globalidad y Ciudadanía | Vol. 7, Núm. 14, junio - diciembre 2021 | ISSN 2395-8448 | http://revpoliticas.uanl.mx/ 
El Derecho a la Ciudad es considerado un derecho humano emergente, esto quiere decir que son derechos humanos nuevos o son reivindicaciones de los ya existentes; estos surgen en respuesta a las intensas transformaciones y la evolución de las sociedades, donde se observan nuevas necesidades humanas u otras necesidades que se han perdido de vista ante la indiferencia de los gobiernos y que es necesario se retomen. Incluso, se debe recordar que en la misma Carta Mundial del Derecho a la Ciudad, en su artículo 1, inciso 2, se establece "El Derecho a la Ciudad es interdependiente de todos los derechos humanos internacionalmente reconocidos, concebidos integralmente, e incluye, por tanto, todos los derechos civiles, políticos, económicos, sociales, culturales y ambientales que ya están reglamentados en los tratados internacionales de derechos humanos" (Carta Mundial por el Derecho a la Ciudad, 2004); así, el Derecho a la Ciudad al ser relativamente reciente, sienta sus bases en los Derechos económicos, sociales y culturales, además incorpora la participación ciudadana y espacio público con una visión integral de los DDHH como factores preponderantes para alcanzar el bienestar y la justicia social. Así pues, la creación del Derecho a la ciudad se basa en el goce de los DESC.

El PIDESC abarca el derecho a un trabajo en condiciones equitativas y satisfactorias (artículo 6 y 7), derecho a la seguridad social (artículo 9) que incluye el derecho al seguro social, derecho a la protección y asistencia de la familia en especial a los niños (artículo 10), derecho a un nivel de vida adecuado, alimentación, vestido, vivienda adecuados (artículo 11), derecho al disfrute del más alto nivel posible de salud física y mental (artículo 12), derecho a la educación (artículo 13), derecho a participar de la vida cultural y derecho a beneficiarse del progreso científico (artículo 15). Es en estos donde radica gran parte de la propuesta realizada por autores como Lefebvre o Soja, quienes establecen como necesaria esa participación cultural, para consolidar la salud y la vivienda adecuada de los habitantes de la urbe.

Dentro de la Carta Mundial por el Derecho a la Ciudad se encuentran prácticamente los mismos derechos que en el PIDESC, aunque es más especifica en ciertos aspectos, por ejemplo, el derecho al “[...] agua potable, energía eléctrica, transporte público y otros servicios sociales [...] "(Carta Mundial por el Derecho a la Ciudad, 2004). Con lo anterior se quiere destacar que el Derecho a la ciudad es el derecho a que se gocen de todos los demás derechos humanos, considerando la mejora continua en las condiciones de vida de las personas; personas que a través del tiempo han ido conquistando el cumplimiento de sus derechos.

No obstante, el Derecho a la Ciudad abarca otros asuntos esenciales para el mejoramiento de la calidad de vida, por ejemplo, el derecho a un hábitat que facilite el tejido de las relaciones sociales, el derecho a sentirse parte de la ciudad (sentido de cohesión social y construcción colectiva), el derecho a 
De la Fuente., M., C., \& Méndez., S., F.

vivir dignamente en la ciudad, el derecho a la convivencia, el derecho al gobierno de la ciudad (Carta Mundial por el Derecho a la Ciudad, 2004).

Aunado a lo anterior y como se comentó en líneas anteriores, dentro del Derecho a la Ciudad se debe realizar un ejercicio pleno de la ciudadanía que significa que todas las personas tienen derecho a "las condiciones necesarias para su realización política, económica, cultural, social y ecológica, asumiendo el deber de la solidaridad" (Carta Mundial por el Derecho a la Ciudad, 2004), tienen derecho a participar en la elaboración y puesta en práctica de las políticas publicas con la finalidad de que estas sean transparentes y exista eficacia en su implementación, en pocas palabras, que sean políticas que de verdad mejoren el nivel de vida de las personas por medio de la disminución de las necesidades más apremiantes y de la utilización adecuada de espacios públicos. Son 3 ejes los principales que se manejan como base en la Carta Mundial por el Derecho a la Ciudad:

El ejercicio pleno de la ciudadanía, es decir el ejercicio de todos los derechos humanos que aseguran el bienestar colectivo de los habitantes y la producción y gestión social del hábitat; la gestión democrática de la ciudad, a través de la participación de la sociedad de forma directa y participativa, en el planeamiento y gobierno de las ciudades, fortaleciendo las administraciones públicas a escala local, así como las organizaciones sociales; la función social de la propiedad y de la ciudad, siendo predominante el bien común sobre el derecho individual de propiedad, lo que implica el uso socialmente justo y ambientalmente sustentable del espacio urbano (Mathivet, 2009).

Los Derechos económicos, sociales y culturales y el Derecho a la Ciudad son simbióticos, es urgente el respeto y goce de todos los derechos que abarcan; siendo necesaria la gestión de prácticas de prevención utilizando todos los recursos disponibles (principio de progresividad).

Asimismo, se deben atender distintas problemáticas y obstáculos como son... llevar a cabo una adecuada armonización legislativa; la adopción de instrumentos internacionales que favorezcan el ejercicio de estos derechos [...] es necesario se garantice que las instalaciones, bienes y servicios públicos se encuentren disponibles y sean accesibles (económicamente), adaptables (deben considerar las diferencias culturales, las barreras lingüísticas, las necesidades específicas e incluso prestar una asistencia adaptada), asequibles (algunos 
pueden ser incluso de acceso gratuito) y de buena calidad. Y se garantice que quienes viven en pobreza puedan acceder a ellos. (CNDH, 2019).

La presidenta de la Comisión de Derechos Humanos del Distrito Federal (CDHDF) explicó que hablar de los DESCA impone pensar en la situación de pobreza en la que viven más de 50 millones de personas en México. Distintos órganos internacionales de supervisión como el Comité DESC y el Grupo de Trabajo sobre el Examen Periódico Universal, ambos del sistema de la Organización de las Naciones Unidas, han manifestado inquietudes sobre las condiciones laborales, la educación, las necesidades de vivienda, las tasas de mortalidad materna, el acceso a la seguridad social, la pobreza y el medio ambiente en México, subrayando los altos índices de población que no gozan de servicios de buena calidad en sus viviendas, que carecen de servicios de protección a la salud o bien que se encuentran en situación de pobreza (CNDH, 2019).

La pobreza en México tiene como factores la insuficiencia del salario mínimo para proporcionar condiciones de vida digna, la alta proporción de trabajo informal, la discriminación en la contratación para personas jóvenes, personas con discapacidad, migrantes, entre otros (CNDH, 2019). Además, la pobreza como fenómeno multidimensional, reproduce patrones de exclusión social y discriminación que transgreden la dignidad de las personas, lo cual se ve reflejado también en las ciudades, en las que el acceso incluso a determinadas zonas y áreas verdes, requieren de un pago.

Articulo II. Fracción 2.2. Los espacios y bienes públicos y privados de la ciudad y de los(as) ciudadanos(as) deben ser utilizados priorizando el interés social, cultural y ambiental. Todos los(as) ciudadanos(as) tienen derecho a participar en la propiedad del territorio urbano dentro de parámetros democráticos, de justicia social y de condiciones ambientales sustentables. En la formulación e implementación de las políticas urbanas se debe promover el uso socialmente justo y ambientalmente equilibrado del espacio y el suelo urbano, en condiciones seguras y con equidad entre los géneros (Carta Mundial por el Derecho a la Ciudad, 2004).

Es importante señalar que hasta el momento la relación de áreas verdes como parte del Derecho a la Salud ha sido poco abordado en relación con su relevancia. Ciertamente han existido algunos estudios que han tenido poco impacto en la mayor parte de las ciudades, especialmente de países en vías de desarrollo. La Organización Mundial de la Salud (OMS) en un estudio realizado por diversos expertos en materia de higiene y de urbanización, señalaba la importancia de que la vivienda al interior de los 
De la Fuente., M., C., \& Méndez., S., F.

espacios urbanos no fuera considerada de manera exclusiva la construcción, ni labor exclusiva de arquitectos e ingenieros, sino de un conjunto más amplio de especialistas que hagan hincapié en las necesidades físicas y psicológicas de los individuos (OMS, 1965, 49).

La OMS señala en relación con las colectividades urbanizadas: "la impresión de soledad (y tal vez monotonía) que se experimenta en ese medio puede dar lugar a estados de tensión nerviosa que conducen a la pasividad o a la delincuencia, a las neurosis o a las enfermedades psicosomáticas" (OMS, 1965, 51), remarcando entre sus principales beneficios la protección contra ruidos, polvo, humo, viento, frío y calor excesivos, entre otros, así como el desarrollo de actividades recreativas. Por tanto, el problema de que la misma colectividad no reclame sus espacios con las respectivas áreas verdes, puede ser un factor determinante en el incremento de la inseguridad. Cabe resaltar que la propuesta de la OMS se desarrolla en el mismo momento histórico que la propuesta de Lefebvre.

La OMS, ha recomendado una extensión de entre doce a dieciséis metros cuadrados de áreas verdes por habitante en las ciudades, con la finalidad de evitar alteraciones de la salud. Por áreas verdes puede entenderse los espacios en donde predomina la vegetación y elementos naturales como lagunas, esteros y senderos no pavimentados (Ministerio de Medio Ambiente de Chile, 2014).

Varios estudios, han avalado en los últimos años que el estrés y las enfermedades mentales tienen una importante relación con la falta de las denominadas áreas verdes, considerando que la depresión y los desórdenes en la salud mental pueden ascender al $15 \%$ de la carga global de enfermedades después del año 2020. Otra problemática es el de la violencia: la tasa de homicidios, por ejemplo, es casi tres veces mayor que el promedio mundial por cada mil habitantes en urbes donde los espacios verdes son escasos (Martínez-Soto, 2016). Otros estudios, apuntan de manera fehaciente a que el establecimiento de mayor número de áreas verdes es un factor preponderante en la baja de actividades criminales y violencia en barrios y colonias, en los que, además, la presencia de árboles promueve una mayor interacción entre vecinos brindando una mayor sensación de comunidad (González).

\section{El Derecho a la Ciudad en el contexto poblano mexicano}

Para hacer referencia del Derecho a la Ciudad en el contexto de Puebla, es menester hacer una breve reflexión sobre el desarrollo histórico de dicha urbe, a fin de contextualizar los retos a los que se enfrenta un Derecho a la Ciudad en este particular punto del globo. 
Puebla de los Ángeles se asentó en el siglo XVI en un valle que originalmente llevaba por nombre Cuetlaxcoapan, que quiere decir "lugar dónde cambian de piel las víboras". El valle se encontraba en ese momento despoblado, factor que fue ideal para los misioneros castellanos de la Orden de San Francisco, ya que era a su vez tabula rasa para una ciudad conforme al pensamiento milenarista de dichos religiosos; sin embargo, es muy importante subrayar que la fundación de Puebla y su elevación a la calidad de ciudad obedecieron a una tendencia jurídica, política y social de la época que fue la relativa a dividir los grupos indígenas de los castellanos (Salazar, 2019,51). Si dicha división y separación se realizó con base a una protección para los naturales, establecida desde la Segunda Audiencia con base en el resultado de la Junta de Burgos o si bien obedeció a cierta tendencia discriminatoria, es hasta hoy punto de controversia entre historiadores indianos.

Puebla fue entonces, fundada como una República de españoles, es decir, solamente los castellanos, mestizos y negros, así como otros grupos mal denominados castas que no pertenecían a los indígenas podían vivir y asentarse en su interior. Por su parte, los indígenas se fueron estableciendo alrededor de la capital angelopolitana desde 1543, a fin de trabajar para los grupos de europeos que vivían dentro de la ciudad y ayudar en la construcción y edificación de la urbe, surgiendo así lo que posteriormente pasarían a ser Barrios como el Alto, en donde se asentaban tlaxcaltecas, Santiago para los cholultecas, San Pablo donde llegaron mexicas, y así otros tantos como Analco y San Baltazar entre otros habitados por indígenas. Cada uno de estos asentamientos podían elegir a sus propias autoridades y alcaldes indios que para el siglo XVI, llegaron a conformar un Cabildo Indígena que era presidido por el Alcalde Mayor de Puebla (Salazar, 2015, 27-28).

Lo anterior fue un factor que contribuyó de manera importante a la estratificación social novohispana conforme a un criterio racial y de nobleza. Cabe señalar que tanto para los europeos como para los indígenas dicha estratificación no era algo extraño, ya que en ambos grupos se había practicado de manera previa a su encuentro durante siglos. Von Humboldt (1966) notó dicha estratificación en el capítulo VI de su obra Ensayo Político sobre el Reyno de la Nueva España.

Ya en la época independiente, se buscó una mayor unanimidad cultural por parte de los líderes del movimiento insurgente, la cual no se obtuvo debido a las diversas corrientes de pensamiento que se conjugaron en el establecimiento de la República Mexicana. Puebla, se caracterizó por un apoyo muy marcado al conservadurismo, y con ello a una consideración muy marcada de las clases sociales. En cuanto a su contexto urbanístico, la Angelópolis se mantuvo durante los siglos XVI al XVII como una importante sede de comercio, sufriendo una decaída importante durante el gobierno de los Borbones. No 
De la Fuente., M., C., \& Méndez., S., F.

obstante, para los albores del siglo XX, Puebla era ya nuevamente una importante sede industrial gracias a las aportaciones del ferrocarril.

Tras el periodo revolucionario, que dejó secuelas económicas de relevancia, la influencia de los hermanos Ávila Camacho impulsó a diversos empresarios, mientras eliminó sistemáticamente a líderes sindicales. Es justamente a partir de la segunda mitad del siglo XX, donde se pueden apreciar con mayor claridad los matices que afectaron lo que en un futuro Lefebvre criticaría al consolidar su concepto del Derecho a la Ciudad. Los gobernadores poblanos comenzaron una serie de reformas en materia urbana reubicando a grupos sociales diversos ya fuera de manera directa o indirecta; ésta última al crear espacios modernos en los que el acceso a aguas y tuberías era posible, dejando antiguas construcciones y edificaciones sin dichos servicios a ser ocupados mediante renta a personas de escasos recursos. Los conflictos resultantes de una serie de desequilibrios económicos y de acceso a servicios, a la manera descrita por Soja, culminaron en explosiones sociales, particularmente en Puebla de grupo de estudiantes, en donde la sangre corrió de manera considerable en aulas y calles (Salazar, 2015).

El establecimiento de empresas tales como Volkswagen e Hylsa derivaron en la creación de fuentes de trabajo, pero también en una innegable dependencia de dichas empresas, alrededor de las cuales se ancló gran parte del desarrollo urbano de Puebla. Situación peculiar fue un incremento en la demografía de la urbe tras el terremoto de 1985, por el cual varias personas se mudaron de la Ciudad de México hacia Puebla. Dos años después la ciudad es declarada patrimonio cultural de la humanidad por parte de la Organización de las Naciones Unidas para la Educación, la Ciencia y la Cultura (Salazar 2015), lo que incrementó su popularidad como destino turístico, pero promovió que ciertos inmuebles donde habitaban personas de escasos recursos, no pudieran arreglarse a menos que se realizara por arquitectos expertos en preservar el patrimonio histórico, lo cual, por el costo de las reparaciones inmuebles provocó que muchos de ellos quedaran abandonados, o que se siga viviendo en los mismos en condiciones de poca salubridad e incluso peligrosas ante los embates de la naturaleza.

No es sino hasta la segunda década del siglo XXI que Puebla considera el Derecho a la Ciudad, el cual comenzó a discutirse de manera un poco tardía en el año 2018, ya que en la Ciudad de México el proyecto se había discutido desde el año 2007, siendo presentado en el 2010 (Carta 2010). En Puebla se presentó finalmente el 30 de octubre de 2019 en medio de un entorno político cuestionable entre la administración estatal y la municipal (Puebla capital 2019). Cabe resaltar que, en ambos casos, las cartas 
de las que se habla siguen siendo un proyecto todavía por terminar de definirse y que ha quedado un poco más en el discurso.

En relación con las áreas verdes como parte del Derecho a la Salud, según el Instituto Nacional de Geografía e Informática (INEGI, 2010) México ha ocupado el onceavo lugar a nivel mundial en número de habitantes, con un total de 112,336,538 personas que se contaron en el año 2010, de las cuales 5,779,829 se encontrarían localizados en el Estado de Puebla, siendo una estadística llamativa que por cada uno que fallece otros cuatro habitantes ocupan su lugar, con un promedio de vida de entre setenta a ochenta años de edad. El Estado de Puebla, cuenta con un total de 34, 251 kilómetros cuadrados de superficie de los cuales solo 1,658 kilómetros pertenecen a áreas urbanas; sin embargo, el mismo INEGI (2010) señala que para el año 2011, el Municipio de Puebla registraba una tasa nula de árboles plantados y de reforestación al interior de la Ciudad.

De igual manera al interior de la Ciudad de Puebla se identifican sólo cuarenta y dos áreas verdes variables que van desde unos cuantos metros cuadrados hasta algunas hectáreas, siendo parches de vegetación aislados, sin corredores verdes y con un alineado de árboles muy escaso, por lo cual no se cumple con la función ambiental, por lo que se requiere del establecimiento de un sistema de gestión de las áreas verdes en Puebla que permita mejorar las condiciones de manejo del arbolado (Santacruz, 2014). En resumen, existen menos de cuatro metros cuadrados de áreas verdes por habitante, porcentaje muy por debajo de los nueve metros cuadrados señalados por la Organización Mundial de la Salud, provocando deterioro en la calidad del aire y de vida de los ciudadanos (Alfaro, 2012).

La planeación urbana en México se basa en los postulados del arquitecto y diseñador urbano Charles-Édouard Jeanneret-Gris, mejor conocido como Le Corbusier, en los cuales se remarca la separación entre usos habitacionales, productivos, de servicios y recreativos y la circulación como principal función urbana, provocando una enorme fragmentación urbana, y que las áreas verdes, fueran fragmentadas, sin escala, forma y siempre implementadas de manera posterior al del desarrollo inmobiliario, es decir primero se construían casas, fábricas y comercios y hasta el final quedaban las áreas verdes incluso de manera accidental. Esto es una nueva ejemplificación de los señalamientos realizados por los diversos teóricos del Derecho a la Ciudad. Iniciando por Lefebvre, adquieren una contextualización real. Primero en las ciudades se ha tendido a conformar el espacio "útil” al necesario para la sociedad en conjunto independientemente del capital, la participación económica, el género, la raza, entre otros. 
De la Fuente., M., C., \& Méndez., S., F.

Los recientes acontecimientos en la capital angelopolitana, entre los que se cuentan las marchas de estudiantes en reclamo a mayor seguridad y resultado de las altas tasas delictivas que imperan no tan sólo en la Ciudad de Puebla, sino en prácticamente toda la entidad, son prueba plausible de que la mayor problemática que se vive actualmente al interior de la sociedad poblana es el de la inseguridad, de la cual se derivan otra gran cantidad de problemáticas y violaciones a los derechos humanos de todas y cada una de las generaciones hasta ahora teóricamente aceptadas. Situaciones como una doble o triple victimización de aquellos que han sufrido un acto delictivo, en especial de grupos vulnerables como son mujeres, niños, indígenas y migrantes en situación irregular, dan fe de los terribles acontecimientos que, en materia de seguridad se vive en Puebla.

Del informe de enero 2020 del Observatorio Ciudadano de Seguridad y Paz, perteneciente al Consejo Ciudadano de Seguridad y Justicia del Estado de Puebla (CCSJEP), se puede determinar que entre homicidios, violaciones, feminicidios y robos con violencia, la Angelópolis es uno de los municipios con más alto índice delictivo de todo el país, lo que a su vez se refleja en abandono de la misma ciudad como espacio público por el temor a ser víctima de un delito y la desconfianza en las mismas autoridades, que deriva también en un círculo vicioso en el que la falta de denuncia incrementa la impunidad (CCSJEP, 2020).

De todo lo anterior, y considerando la siempre interdependencia entre los Derechos Humanos, es necesario señalar que la inseguridad coarta a su vez la libertad física de la persona, además de que, a niveles institucionales, se observa a veces un preocupante decremento de la libertad de expresión, especialmente cuando se busca que no se sepan los verdaderos números que de la incidencia delictiva existen, derivando en informes contradictorios sobre el tamaño del problema.

A nivel estatal, los principales problemas se encuentran en los municipios de Tehuacán, Huauchinango, San Martín Texmelucan y la misma ciudad capital (Proyecto Social Ciudadano, 2020). Cabe señalar que los principales grupos vulnerables son las mujeres, con altas tasas de violaciones y feminicidios de los cuáles el verdadero porcentaje es desconocido, ya que se sabe que en muchas ocasiones no se aplica el Protocolo de Investigación con perspectiva de Género, aplicando a las mujeres y niñas una violencia institucional con ello. Nada más al interior de la capital, los principales focos de alerta se ubican en Barrio de Santiago, Centro Histórico, San Manuel, Guadalupe Hidalgo, La Paz, Buenavista, Santa María, Bosques de San Sebastián, San Pablo Xochimehuacan y La Libertad, puntos 
urbanos que desgraciadamente en las últimas semanas han centrado la atención pública (Secretaría de seguridad Pública y Tránsito Municipal, 2020).

Es necesario la aplicación de un protocolo que coadyuve en desarrollar un verdadero Derecho a la Ciudad en Puebla, para su realización es esencial dividir en dos etapas el desarrollo, la primera de indagación, evaluación y análisis, seguida de la instrumentación de actividades que permitan reconocer y aplicar un derecho a la ciudad conforme a las verdaderas necesidades de la población. En la etapa de indagación e investigación se debe recurrir a un método dialéctico, en el que se plantee como tesis la actual situación de la ciudad bajo tres aspectos principales:

Comunidad. Con especial énfasis en Juntas auxiliares, barrios y finalmente colonias; historia desarrollo, tendencias a la unidad como grupo particular serán relevantes. Se deben buscar conectores de dichas comunidades con acciones de responsabilidad cívica, verificando denuncia y acciones de protección conjunta, así como el éxito de estos últimos y el grado en que dichas acciones violentan el marco normativo existente o los derechos de terceros. Un factor para considerar de manera secundaria es la injerencia de programas por parte de Empresas Socialmente responsables.

Delincuencia. Se debe solicitar el apoyo de Organismos de participación ciudadana como el CCSJEP y en vinculación con Universidades realizar un análisis científico de la realidad de los delitos de trata de personas, secuestro y feminicidio, alejado de intereses políticos o posiciones partidistas.

Factores ambientales. Con especial énfasis en el desarrollo de áreas verdes y espacios con posibilidad de ser recuperados por la comunidad para el esparcimiento. De igual manera es muy importante verificar el acceso al agua y relacionar todo lo anterior con grupos de edades y la identificación de estos con los espacios.

En la segunda etapa, la instrumentación debe consistir en plantear la antítesis de los problemas ubicados en la etapa previa, mediante el reforzamiento mediante talleres a las comunidades de tres aspectos: la recuperación de espacios públicos y el incremento de áreas verdes, la protección comunitaria ante la delincuencia y la responsabilidad cívica en el que se fomente la cultura de la denuncia haciendo uso de los muchos instrumentos a distancia que actualmente existen pero que se desconocen. 


\section{3.- MÉTODO}

Tal como se señaló en la introducción, el presente estudio utilizó una metodología, inductiva, histórica y sistémica, con base en un iuspositivismo. La técnica desarrollada fue exclusivamente documental. El presente trabajo es una primera aproximación teórica al problema, cuyas aplicaciones prácticas se analizarán en un momento posterior.

\section{4.- CONCLUSIONES}

En la actualidad no es posible señalar todavía una ciencia analítica de la ciudad; sin embargo, para formar la misma se requiere de la práctica social reflejada en los esfuerzos de la colectividad. Para Lefebvre, la ciencia de la ciudad toma prestados sus métodos, enfoques y conceptos de las ciencias a las que denomina "fragmentarias", es decir, las ciencias sociales que analizan de manera separada la realidad social y humana, pero señalando que debe realizarse mediante una sistematización y programación estratégica que considere a la ciudad como una realidad todavía no consumada y que ya ha superado a la "ciudad histórica”. Para ello es necesario analizarla, dividirla en sus partes y volver armarla, estudiando la misma más allá de un "objeto de consumo cultural" en el que lo "urbano" permanece en un estado de constante actualización y mutación (Kofman, Lebas y Lefebvre, 1996). Pero Lefebvre también advierte un grave problema en la cotidianeidad de la ciudad, y es que desde el siglo pasado la ausencia del humanismo clásico se encuentra más presente, abrazando un nihilismo en el que la cruel frase de que "Dios ha muerto", pareciera tomar el alma de los miembros de la sociedad. Por tal motivo, el autor en comento remarca la necesidad de reforzar el humanismo.

Es en las aportaciones de Soja y Mele donde se puede ubicar el elemento diferenciador de la propuesta puramente humanista ( $\mathrm{y}$ anticapitalista) de Lefebvre, al señalar dos elementos básicos para instrumentar un derecho a la ciudad: La educación y las pequeñas comunidades. Es en este punto donde las grandes urbes han perdido la sensación de comunidad que otrora se tenían. El reforzamiento de dichos lazos a través de fomentarlos en los barrios y colonias puede coadyuvar, pero para ello es necesario programas en los cuales las mismas comunidades reclamen las áreas verdes, las cuiden y la protejan en conjunto. Pero para ello es necesario que las universidades, por ejemplo, realicen programas de orientación a vecinos de las áreas, partiendo que los mismos estudiantes se tornen parte de los medios sociales y urbanos con los que conviven y aportando algo más desde la perspectiva de cada disciplina en la que se forman.

100 Revista Política, Globalidad y Ciudadanía | Vol. 7, Núm. 14, junio - diciembre 2021 | ISSN 2395-8448 | http://revpoliticas.uanl.mx/ 
De la Fuente., M., C., \& Méndez., S., F.

Con esto en mente, se podrá reforzar la militancia urbana que atienden los teóricos del Derecho a la Ciudad, incrementando una idea de civismo y de responsabilidad para con el entorno. De ahí, se podrá incluso establecer con ese sentido de comunidad, teatros, mercados y hortalizas urbanas que sean el sustento de una mejora en la calidad de vida, al menos en los ambientes donde se vive, disminuyendo la delincuencia y la violencia y permitiendo una mejor calidad de vida en ambientes mucho más limpios y agradables.

\section{REFERENCIAS}

Alfaro Alfaro, A. (21 de diciembre, 2012). Registra Puebla un déficit en Áreas Verdes (La Jornada de Oriente). http://goo.gl/zlKm4U

Comisión Nacional de Derechos Humanos. (2019). Informe anual de actividades 2019. http://informe.cndh.org.mx/menu.aspx?id=40072

Consejo Ciudadano de Seguridad y Justicia del Estado de Puebla - OCSEPAZ. (2020). Informe de Incidencia Delictiva. Puebla: Autor. https://consejociudadanopuebla.org/download/informe-deincidencia-delictiva-municipio-de-puebla-septiembre-2020/

Costes, L. (2014). Neoliberalization and Evolution of the "Right to the City" Justice Spatiale, 6 (4), 1 11. https://www.jssj.org/wp-content/uploads/2014/06/Costes-Eng-n\%C2\%B06-jssj.pdf

Foro Social de las Américas. (2004) Carta Mundial por el Derecho a la Ciudad. https://www.ugr.es/ revpaz/documentacion/rpc_n5_2012_doc1.pdf

González, V. (s/f). En los barrios más verdes hay menos delincuencia (Muy Interesante). https://www.muyinteresante.es/naturaleza/articulo/en-los-barrios-mas-verdes-hay-menosdelincuencia-281470640165

Gutiérrez, B. (19 de octubre, 2016). El derecho a la ciudad como práctica ciudadana (Democracia abierta). https://www.opendemocracy.net/es/democraciaabierta-es/el-derecho-la-ciudad-comopr-ctica-ciudadana/

Habitat International Coalition América Latina. (2011). Carta de la Ciudad de México por el Derecho a la Ciudad. https://hic-al.org/wp-content/uploads/2019/02/CARTA_CIUDAD_2011$\underline{\text { muestra.pdf }}$

Harvey, D. (2008). El Derecho a la Ciudad. New Left Review, nov-dic (53), 23-39. https://newleftreview.es/issues/53

Humboldt, A. (1966). Ensayo Político sobre el Reino de la Nueva España. México: Porrúa. 
De la Fuente., M., C., \& Méndez., S., F.

Instituto Nacional de Estadística Geografía e Informática. (2010). México en Cifras. http://www3.inegi.org.mx/sistemas/mexicocifras/default.aspx

Kofman E.; Lebas, E. y Lefebvre, H. (1996). The Right to the City (The Anarchist Library). https://theanarchistlibrary.org/library/henri-lefebvre-right-to-the-city\#toc1

Martínez-Soto, J.; Montero López-Lena, M. y De la Roca Chiapas, J. (2016). Efectos Psicoambientales de las Áreas Verdes en la Salud Mental. Interamerican Journal of Psychology, 50 (2), 204-2014. Mathivet, Ch. (2009). El derecho a la ciudad: claves para entender la propuesta de crear otra ciudad posible. http://base.d-p-h.info/es/fiches/dph/fiche-dph-8034.html

Mele, P. (2016). ¿Qué producen los conflictos urbanos? En Carrión Fernando y Erazo Jaime (Coord.). El derecho a la ciudad en América Latina, México: UNAM.

Ministerio de Medio Ambiente de Chile. (2014). Áreas Verdes. http://www.mma.gob.cl/1304/articles52016_Capitulo_6.pdf

Molano Camargo, F. (2016). El derecho a la ciudad: de Henri Lefebvre a los análisis sobre la ciudad capitalista contemporánea. Folios, 44. 3-19.

Oficina del Alto Comisionado de las Naciones Unidas para los Derechos Humanos. (1966). Pacto Internacional de los Derechos Económicos, Sociales y Culturales. https://www.ohchr.org/sp/professionalinterest/pages/cescr.aspx

Pueblacapital. (2019). Presentan Carta Poblana por el Derecho a la Ciudad. Pueblacapital, 30 de octubre, https://bit.ly/2x6zT59

Salazar, J. (2015). La invención de un sueño. Historia de la Ciudad de Puebla de Ángeles. México: Tirant Humanidades.

Salazar, J.; Ortiz, L. y Méndez, F. (2019). "Factores históricos de la discriminación hacia las personas indígenas en el sistema jurídico y social mexicano”. En La justicia Penal Indígena en México, coordinado por Guzmán Héctor, Sierra María Teresa y Velázquez Jeannette. México: Ubijus.

Secretaría de Seguridad Pública y Tránsito Municipal de Puebla. (2019). Mapa delictivo. $\underline{\text { http://bit.ly/3cBG7tC }}$

Proyecto Social Ciudadano (2020). Semáforo Delictivo Puebla. http://puebla.semaforo.com.mx/

Soja, E. (2014). En busca de la justicia espacial. España: Tirant LoBlanch Humanidades.

United Cities and Local Goverments - UCLG. (2014). Derecho a la ciudad: qué es, de dónde surge y a quién va dirigido. https://www.uclg.org/en/media/news/derecho-la-ciudad-que-es-de-dondesurge-y-quien-va-dirigido

102 Revista Política, Globalidad y Ciudadanía | Vol. 7, Núm. 14, junio - diciembre 2021 | ISSN 2395-8448 | http://revpoliticas.uanl.mx/ 